\title{
Early occurrence of brief potentially ictal rhythmic discharges [B(I)RDs], and subsequent emergence of asymmetric extreme delta brush (EDB) in Anti-NMDA receptors encephalitis
}

Ocorrência precoce de breves, rítmicas e potencialmente ictais descargas [B(I)RDS], e surgimento posterior de assimétrico extremo "delta brush" (EDB) num caso de encefalite Anti-NMDAr

Valmir Passareli',2, Lais Medeiros², Mateus Simabukuro', Meire Baldocci², Sonia Brucki², Maria Sheila Guimarães Rocha ${ }^{2}$

A 12 years-old boy was admitted in coma without motor deficit, with normal CT scan, CSF with lymphocytosis, and EEG showing B(I)RDS (Figure A). Continuous EEG (24 hours) and subsequent serial intermittent EEGs were performed. Seizures were not documented. Patient developed orofacial diskynesia and coreoathetosis movements affecting superior limbs. Later, EEG showed asymmetric EDB pattern (Figure B). MRi was unremarkable.
After immunotherapy, his level of consciousness improved. Anti-NMDAr antibodies were detected in serum and CSF.

$\mathrm{B}(\mathrm{I}) \mathrm{RDS}$ were described in critically ill patients, often associated with brain injury and seizures, but no reported in Anti-NMDAr encephalitis ${ }^{1}$. EDB has been described in AntiNMDAr encephalitis; however, their clinical significance remains to be determined ${ }^{2}$.

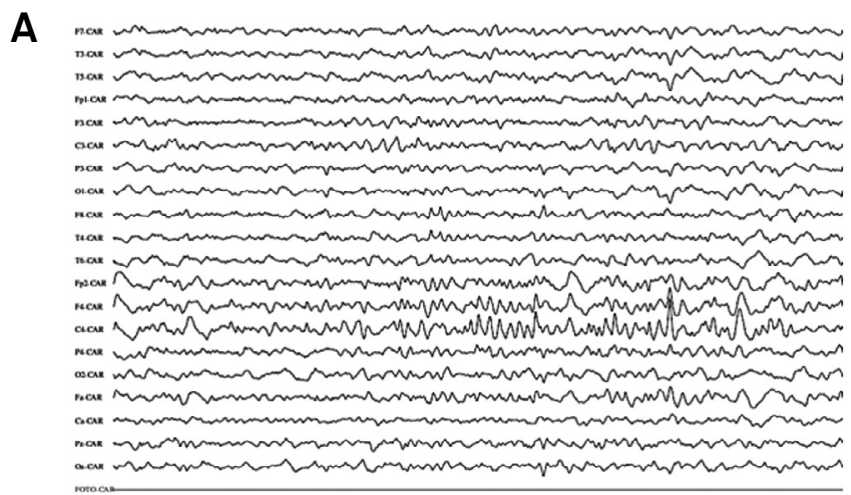

B

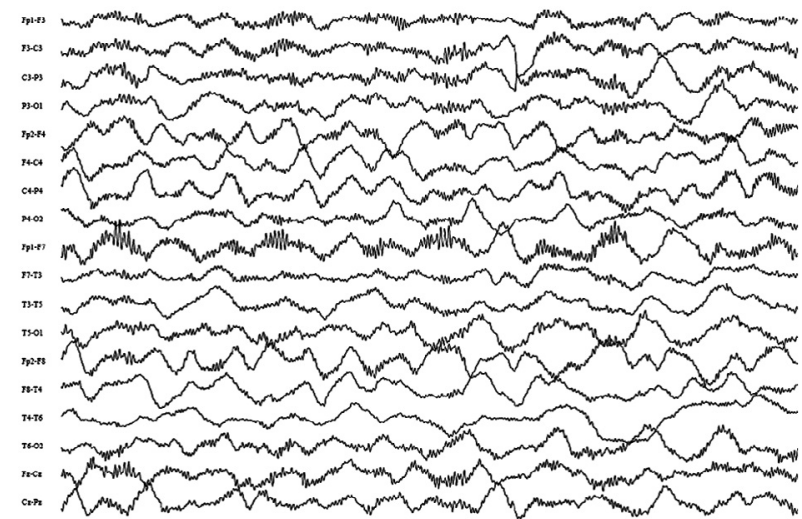

Figure. (A) First electroencephalographic record showing frontocentral (F4, C4 electrodes) burst of rhythmic activity with a frequency of 12-14 Hz, during 06s, a Brief potentially ictal rhythmic discharges - B(I)RDS pattern. (B) EEG eight days after admission, showing asymmetric extreme delta brush (EDB) with delta activity more pronounced in right hemisphere. Parameters were as follows: sensitivity $7 \mu \mathrm{V} / \mathrm{mm}$, time constant $0.3 \mathrm{~s}$ and high-frequency filter $70 \mathrm{~Hz}$.

\section{References}

Yoo JY, Rampal N, Petroff OA, Hirsch LJ, Gaspard N. Brief potentially ictal rhythmic discharges in critically ill adults. JAMA Neurol. 2014;71(4):454-62. doi:10.1001/jamaneurol.2013.6238
2. Schmitt SE, Pargeon K, Frechette ES, Hirsch LJ, Dalmau J, Friedman D. Extreme delta brush: a unique EEG pattern in adults with anti-NMDA receptor encephalitis. Neurology. 2012;79(11):1094-100. doi:10.1212/WNL.0b013e3182698cd8

'Universidade de São Paulo, Faculdade de Medicina, Hospital das Clínicas, Departamento de Neurologia, Sao Paulo SP, Brazil;

${ }^{2}$ Hospital Santa Marcelina, Departamento de Neurologia, Sao Paulo SP, Brazil.

Correspondence:Valmir Passareli; HCFMUSP, Departamento de Neurologia; Rua Doutor Eneas de Carvalho Aguiar, s/n; 05409-010 São Paulo SP, Brazil; E-mail:valmirpas@ig.com.br

Conflict of interest: There is no conflict of interest to declare.

Received 08 July 2015; Received in final form 13 August 2015; Accepted 02 September 2015. 